\title{
The Effect of Cognitive Dissonance on Perceptions of Emotion Jacqueline King ${ }^{1}$ and Mengfei Cai ${ }^{{ }^{*}}$
}

${ }^{1}$ James Madison University, Virginia, United States

${ }^{2}$ Manhattanville College, New York, United States

\begin{abstract}
The influence of display rules and social norms can interfere with individuals' abilities to express themselves, other's abilities to acknowledge their own perceptions and empathetic responses to others in emotionally charged situations. This phenomenon creates inconsistencies in what one believes and what one can outwardly acknowledge, potentially inducing an experience of cognitive dissonance. The presence of cognitive dissonance is broadly defined by the presence of inconsistencies among thoughts, beliefs, and behaviors and is frequently studied in terms of dissonance-alleviating responses. The current study explores the potential dissonance-alleviating responses of being forced to outwardly accept a particular connotation of an image that is not inwardly perceived. Participants $(n=30)$ were randomly assigned to either a congruency/control condition or a non-congruency/dissonance condition. The two groups were compared post-dissonance or post-control priming based on their emotional acuteness, as measured by the Geneva Emotion Recognition Test (GERT-S). The results of an independent t-test indicate a statistically significant difference between the non-congruency/dissonance condition $(M=$ 22.87, $S D=4.91)$ and the congruency/control condition $(M=27.2, S D=3.95)$ on the GERT-S measure, $t(28)=-2.67, p=.013$, with an effect size of $\hat{\mathrm{d}}=0.21$, indicating a small effect between groups.
\end{abstract}

\author{
Publication History: \\ Received: January 27, 2021 \\ Accepted: April 10, 2021 \\ Published: April 12, 2021
}

\section{Keywords:}

Cognitive dissonance, Display rules, Emotion, Emotion recognition, Empathy, Social norms

\section{Introduction \\ Background \\ Every society or culture will eventually demand its inhabitants conform to certain societal norms. The term "norms" sociologically refers to the role of values on behavior [1]. In accordance with the social norms theory; individuals will eventually experience a pressure \\ the shock of the earthquake, but were spared the severity, began to spread rumors that an even worse earthquake was to come. Festinger explained this in terms of what he would later call, insufficient justification [8]-i.e.the people who had been spared disaster had worked themselves into such a frenzy before the earthquake's arrival, that the benign shocks left them with insufficient justification for the intense fear they had been feeling. Therefore, those people spread rumors to create a physical sense of danger that justified their sense of fear [9].} to act in the ways their society has deemed appropriate [2]. One specific example of norms pressure is emotional regulation. While emotional regulation can take the form of reappraisal, which allows one to reframe potentially emotionally charged situations, it can frequently occur via suppression, involving conscious or subconscious efforts to inhibit emotion-related expressions [3]. While this is often discussed with regards to gender differences [3-5], some degree of emotional regulation is expected in everyday situations. For example, in various cultures, crying might be deemed socially unacceptable in many social situations but as acceptable in others. Asking, "How are you doing?" could intended as a mere social nicety, or as a genuine question as to someone's personal well-being. Because of these social norms, individuals are expected to mask their true feelings in many social contexts, and others may be incentivized to ignore signs that "fine" doesn't actually mean "fine".

The resulting question is whether this experience yields negative effects on individuals or how they relate to others. In the current study, this question is investigated within the framework of cognitive dissonance [6]. Inconsistencies in perceptions regarding an emotionally imbued event, and the lack of acknowledgement that is societally fostered, are explored via the possible dissonancealleviating action of reducing our perceptions of others.

\section{Literature review}

\section{Festinger's cognitive dissonance}

Festinger's [6] theory of cognitive dissonance initially built upon the work of Prasad [7], who observed curious reactions from people in India following a severe earthquake. Individuals who had experienced
Cognitive dissonance theory hinges on the notion that individuals have a need to keep their cognitions, beliefs, and behaviors in line with one another; and inconsistencies can create a cognitive discomfort that people will try to alleviate [6]. This definition allows the theory to be applicable in a wide variety of research applications investigating intrapersonal inconsistencies. Festinger [6] specifically outlined three applicational situations-post-decisional dissonance, effort justification, and insufficient justification-which have gone on to be widely studied by the research community with regards to their influences on various aspects of life and decision making [1013].While each of Festinger's proposed experiences have their own unique definitions, they also tend to be researched in terms of what can be deemed dissonance-alleviating responses-which have typically included some sort of shift in an individual's perceptions and beliefs surrounding different decisions, events, and experiences [6].

\section{Post-decisional and effort justification responses}

The identification and classification of cognitive dissonance often has centered around the presence of a dissonance-alleviating response. In the case of post-decisional dissonance, a response arises from the

"Corresponding Author: Dr Mengfei Cai, Manhattanville College, 2900 Purchase St, Purchase, NY 10577, United States; E-mail: Mengfei.Cai@mville.edu

Citation: King J, Cai M (2021) The Effect of Cognitive Dissonance on Perceptions of Emotion. Int J Psychol Behav Anal 7: 175. doi: https://doi.org/10.15344/2455$3867 / 2021 / 175$

Copyright: (C) $2021 \mathrm{King}$ et al. This is an open-access article distributed under the terms of the Creative Commons Attribution License, which permits unrestricted use, distribution, and reproduction in any medium, provided the original author and source are credited. 
experience of having to choose between two comparable options. To alleviate discomfort stemming from fear one may have "chosen wrong", individuals tend to reframe the situation in their minds postdecision, in a way that makes the chosen option appear clearly superior to the alternative-even if the two options were equal in functionality [6]. Effort justification refers to the dissonance one experiences when unpleasant tasks must be undertaken to yield desirable outcomes. Such dissonant thoughts commonly center around the question "Was it worth it?", in which individuals who are working harder for a desirable effect have a cognitive drive to justify their efforts. This, in turn, leads to increased satisfaction in those working harder, as they attempt to justify increased effort by emphasizing those efforts' successes [6]. Responses to insufficient justification, however, are less straightforward than that of the corresponding types, as the range of situations in which individuals experience it is much broader, thus can, and has, yielded a myriad of different responses [7,6].

\section{Insufficient justification responses}

Insufficient justification is slightly less intuitive than the other two applications; however, it ties directly into the definition outlined above for cognitive dissonance. It has already been shown how insufficient justification could be applied to explain the earthquake phenomenon; however, its originating experiment was far more complex. Festinger and Carlsmith [8] designed an experiment that created dissonance in participants' beliefs regarding a tedious experiment by paying different amounts (\$1 and \$20 groups) to subjects in each group to report that the experiment was interesting. The important finding was that, contrary to the expected, the group that was paid less money later reported having enjoyed the experiment more, that they found the experiment to be interesting, and showed a desire to repeat it. Meanwhile, the group who was paid the $\$ 20$ had no trouble admitting it was boring later on and did not seem to be bothered by the lie. Festinger and Carlsmith explained this in terms of "sufficient justification" [8]. The payment made in the $\$ 20$ group was deemed sufficient justification for the lie, and thus individuals did not experience dissonance when reporting on the experiment. On the other hand, the group that was paid $\$ 1$ for their description of the experiment was not able to justify their behavior for the meager reward and thus, because their behavior contradicted their beliefs, they simply altered their belief that the experiment had been boring to alleviate the discomfort (dissonance).

\section{Belief vs. Behavior}

The results of the experiment that introduced insufficient justification [8] differ slightly from those of the original earthquake example [7] in terms of severity and consequences, but they both highlight an obvious and key element of internal response to dissonant experiences. That is, in the case of dissonance between belief and behaviors, one has two clear options-changes their beliefs or changes their behaviors. Frequently, changing behaviors is not a viable option once a decision has been made, effort has been exerted, or when an event has already transpired. However, in the cases where it is an option, dissonance has been shown to have different effects if one believes a decision is reversible [14]. Still, more often with dissonance the resulting shift occurs in beliefs-with the examples of post-decisional reframing or effort justification, and their effects on various sects of life, such as with regards to policy attitudes [10], voting behaviors $[15,11]$ education [13], or weight loss and psychotherapy [12]. This shift in belief is what can be seen in Festinger and Carlsmith's initial experiment-individuals in the $\$ 1$ group changed their belief about the experiment to alleviate dissonance related discomfort [8]. However, the observations of Prasad [7] differed in terms of the temporal reality being affected by the dissonance-where Festinger's experiments have all yielded the reframing or denial of past events $[8,6]$, Prasad showed how people can also respond to dissonance in the continual denial of a reality-even going so far as to blatantly reject that current reality as is shown in the example of rumors, serving as attempts to control/ change an outward environment to be congruent with one's own inward experiences.

\section{Modern interpretations}

Since cognitive dissonance can be applied to many situations in which inconsistencies in cognitions, thoughts, beliefs, behaviors, etc. exist, others have utilized the theory to explain phenomena outside the realm of Festinger's initial three effects. For example, various researchers have attempted to broaden the scope of dissonance by looking at how simplistic a cognitive inconsistency can be and still induce cognitive discomfort. Findings support the notion that any cognitively held inconsistency, no matter how small, has the potential of inducing dissonant states within the brain $[16,17]$. Other research has attempted to narrow down the theory by looking at the specific instances in which dissonance theory holds true and when it doesn't. One such attempt was noted earlier with relation to post-decisional dissonance, as the separation of irreversible and reversable decisions was made [14]. Other attempts look at personality traits as risk factors for cognitive dissonance [18] or examine how cognitive dissonance is experienced differently across cultures $[19,20]$.

\section{Social norms}

The relationship between cognitive dissonance and social norms may not seem entirely obvious at first, but because social norms often influence individual's behavior in various situations, it can frequently be the cause of dissonance inducing behaviors. The common example of the "white lie" can be examined here, as one might hold the internal belief that lying is wrong but feel pressured to conform to social expectations to maintain a social balance. From a cognitive dissonance standpoint, an individual who has engaged in such a behavior has few options to alleviate the dissonant discomfort. Assuming the lie was about something as basic as liking a haircut, their only options would be to come clean or based on the insufficient justification principle, be forced to change their appraisal and believe they actually did like the haircut. It can also be argued that depending on the person, and their values, it's possible they may not experience cognitive dissonance at all if they view social expectations and norms as an adequate justification for their behavior.

\section{Display rules}

Social norms can often dictate what are known as display rules. Display rules were originally introduced by Paul Ekman and Friesen [21]. Based on Ekman's background in cross-cultural non-verbal behaviors and micro-expressions, display rules were initially discussed in terms of emotional displays, as they dictate the situations in which individuals are "allowed" to display their emotions in a socially acceptable way. Since then, emotion seems to have remained the main focus of display rules [22-25]. Obviously, because display rules are often learned by socialization factors and social norm internalization, several differences have been noted across culture. One prominent distinction was made between individualistic and collectivist cultures-research has found cross-cultural display effects for in-group vs. out-group behaviors, but expressivity widely varied [26]. Likewise, research conducted regarding smaller cultural contexts, such as 
work atmospheres have found a significant emphasis on display rules [27]. This makes intuitive sense as professional environments demand professionalism, which might consequently limit emotional expressions. Additionally, such rules can disincentivize individuals from interacting with any emotional contexts in certain settings, be it their own feelings or their perceptions of others.

\section{Gender differences and emotion regulation}

In terms of emotional expression, there is clear evidence that society places different standards on men and women, which can cause harm to both genders. For example, women are often deemed so emotional that it has become a stereotype and a means of discreditation. Yet socially, this means women are allowed to express their emotions more openly than men, who have traditionally had the expectation of stoicism $[28,29]$. These differences are also thought to have led to different systems of emotional regulation-while both sexes have been shown to engage in the simple tactic of suppression, women have been shown more capable of emotional reappraisal compared to men [30]. Interestingly, unhealthy emotional regulation can be a learnedtechnique in childhood, as children have been shown to internalize the mechanisms being used by their parents [31].

\section{Reductions in empathy}

Research has examined the different reasons individuals might veer away from empathetic responses. Emotional regulation has been discussed as one such possibility, under the assumption suppressing our own emotions makes us less willing to interact with others' [32]. Additionally, cognitive costs have been explored, as researchers take a cognitive toll approach to explain why individuals might choose to avoid empathy on the premise that it is a cognitively exhausting experience [33,34]. However, no known research has considered the possibility that empathy reduction can occur as a result of cognitive dissonance. The notion that social norms can prevent one from interacting with their own and others' emotions could potentially cause dissonant cognitions in one's perception of an emotional situation. Thus, empathy reduction could serve as the dissonance alleviating mechanism when inconsistencies cannot be otherwise addressed. The current study explores empathy in terms of emotionalintelligencei.e, the acuteness of one individual to the emotional experience of another.

\section{Study overview}

The current study builds upon the premises of social norms and display rules as they affect emotion regulation in attempt to understand additional consequences the practices might have. A cognitive dissonance framework is used to explore the relationship between emotional incongruencies and individual perceptions of others in emotional contexts. It is hypothesized that cognitive dissonance effects (as induced by images of a specific valence and the presentation of words opposing that valence) will yield attempts to alleviate dissonance related distress by a reduction in participants' sensitivity to the emotions of others, as measured by the Geneva Emotion Recognition Test (GERT-S).

\section{Methods}

\section{Participants}

Participants in this study were 30 college students over the age of 18. In total, $77 \%$ of the participants identified as female $(n=23)$ and $23 \%$ identified as male $(n=7)$. Participants were recruited via email announcements from professors, some with the offer of extra credit, and all with the incentive of being entered into a drawing to win a $\$ 20$ gift card. Each participant was randomly assigned via an online generator to one of two groups-either a congruency condition or a non-congruency condition. The ratio of male to female was relatively consistent with 4 males in the control condition, and 3 males in the dissonance condition.

\section{Materials}

Materials used in this experiment included the online software Typeform, used to display images from the Geneva Affective Picture Database (GAPED) (Appendix A \& B) Supplementary files and words of corresponding or opposing valences to those of the images (Appendix C) Supplementary files. Additionally, the short version of the Geneva Emotion Recognition Test (GERT-S) was applied (Appendix D) Supplementary files. The GERT-S consists of 42 short video clips with sound (duration 1-3s), in which ten professional French-Swiss actors (five male, five female) express 14 different emotions. After each clip, participants are asked to choose which of the 14 emotions best describes the emotion the actor intended to express. Responses are scored either correct (1) or incorrect (0), yielding a total average GERT-S score that can range from 0 to 1 [35]. All measures were completed on a computer using either headphones or speakers.

\section{Procedure}

Participants were first randomly assigned into two conditions: a congruency condition and a non-congruency condition. Both conditions included two phases. In phase one, individuals in the congruency conditions viewed multiple emotionally charged images, and chose from four emotionally charged words, as to which one was most fitting to the overall tone of the image. Several options were satisfactory, and there was the addition of an "Other" option, in which participants were able to write in whatever word they chose to describe the image (Appendix A).

Individuals in the non-congruency group viewed the same images. However, all options opposed the image's corresponding valence and connotation, and there was no option for write in (Appendix B). This phase exists to create a dissonant state in those in the non-congruency condition by forcing participants to pick an attribution they don't actually believe to be representative. Words were chosen based on research defining their arousal and valence and common evaluation of positive or negative connotations as determined by the researcher.

In the second phase of the experiment, both the congruency and non-congruency groups completed the short version of the Geneva Emotion Recognition Test (GERT-S), a forty-two-question measure testing individual's emotional acuteness by means of emotion recognition.

To manage the expectations of both groups, a deceptive informed consent form was collected at the beginning of the protocol to prevent any from guessing the true nature of the research and already noting the researcher's expectations on performance with the GERT-S scale. Upon completing the protocol, participants received and signed a debriefing form, with the option to withdraw from the study if they so chose. Finally, demographic information was collected. The protocol was reviewed and approved by the Institutional Research Review Board of this college located in West Virginia. All subjects were provided with informed consent, debriefed, as well as provided 
resources to the counseling center should any participants experience psychological discomfort. Data was analyzed using an independent $t$ test to compare the two groups, with a significance level of .05.

It should be noted that the experiment was not able to remain consistent throughout its duration due to the impact of the COVID-19 pandemic, which required the second half of data collection to occur in online formats, as opposed to in the lab. Therefore, there was a lower level of variable control in the second half of the experiment. The significance of the shift is discussed below.

\section{Results}

An independent t-test was used to test the significance of the difference between scores on the GERT-S for those in the dissonance condition and those in the control condition. Descriptive statistics are presented in Table 1 . Assumption of homogeneity of variance was satisfied, Brown-Forsythe $F(1,28)=.359, p=.554$, as well as the assumption of normality for both conditions (Table 2). All other assumptions were met. Results of the independent $t$-test indicate a significant difference between groups, with the dissonance condition $(M=22.87, S D=4.91)$ performing significantly worse on emotion recognition via the GERT-S than did the control condition $(M=$ $27.2, S D=3.95), t(28)=-2.67, p=.013$, with a magnitude of $\hat{\mathrm{d}}=0.21$, indicating a small effect size.

\begin{tabular}{|c|c|c|c|c|c|c|}
\hline & $\mathrm{N}$ & Mean & \multicolumn{2}{|c|}{ Standard Deviation } & Min & Max \\
\hline Dissonance & 15 & 22.87 & \multicolumn{2}{|c|}{4.91} & 13 & 30 \\
\hline Control & 15 & 27.28 & \multicolumn{2}{|l|}{3.95} & 21 & 32 \\
\hline \multicolumn{7}{|c|}{ Table 1: GERT-S scores by Condition. } \\
\hline \multicolumn{2}{|l|}{ Condition } & \multicolumn{2}{|c|}{ Shapiro-Wilk $W$} & $d f$ & \multicolumn{2}{|l|}{$p$} \\
\hline \multicolumn{2}{|l|}{ Dissonance } & \multicolumn{2}{|l|}{.960} & 15 & \multicolumn{2}{|l|}{.693} \\
\hline \multicolumn{2}{|l|}{ Control } & \multicolumn{2}{|l|}{.884} & 15 & \multicolumn{2}{|l|}{.054} \\
\hline
\end{tabular}

Table 2: Test of normality of GERT-S scores for each condition.

Additional inquiries were conducted to look at gender differences and to investigate potential effects of switching to an online format on results. With regards to gender, there did not appear to be any practical differences in emotion recognition scores between males $(M=25.42$, $\mathrm{SD}=4.96)$ and females $(\mathrm{M}=24.91, \mathrm{SD}=4.99)$. However, due to the small sample size, significance was not tested here. There was not a significant difference in the shift between in-person administration $(\mathrm{M}=24.93, \mathrm{SD}=4.63)$ and online administration $(\mathrm{M}=25.13, \mathrm{SD}$ $=5.31), \mathrm{t}(28)=-0.11, \mathrm{p}=.913$ that resulted due to the impact of COVID-19 on data collection.

\section{Discussion}

The results of the t-tests indicate there was a significant difference between the two groups. These results support the proposed hypothesis that dissonant conditions can interfere with emotion recognition. Yet, the effect size was small, meaning any dissonance experience may not interfere to a great extent. Results of the t-test examining the impact in changes to administration setting indicated that the shift in administration technique did not affect results. Lastly, results of this study did not support commonly found gender differences in emotion recognition. Implications of these findings are discussed below.

\section{Implications}

The definitional applicability of cognitive dissonance makes it a useful tool to explore various cognitive incongruencies. The current research contributes to the literature by providing preliminary findings indicating that dissonance may interfere with emotional acuity/recognition. Here, emotional recognition is investigated as a potential reflection of empathy. Therefore, future steps would be to explore additional facets of empathy, such as using measures to evaluate if the effect holds on other forms of empathy, like emotional empathy or cognitive empathy [36].

There could also be implications of the social norms that prevent individuals from outwardly acknowledging the emotions of others. To give an example, social norms dictate a difference between the experience of observing the emotions of one with which we have a close relationship and observing the emotions of a colleague or acquaintance. When one perceives that a friend is experiencing emotional distress, there are channels in which that observation can be outwardly acknowledged. For instance, we can check in on them, ask them how they're doing, etc., hopefully prompting some sort of resolution in which any possible confusion is resolved or our perceptions are validated and a discussion emerges to help the individual. However, when we perceive a mere acquaintance is experiencing a similar level of emotional turmoil, it is not always deemed appropriate to pry into their emotional state. Thus, there may not be a true resolution to our observations nor justification for any concern. This study supports the notion that such practices inducing incongruency between outward acknowledgement and inward beliefs could have maladaptive effects, causing individuals to withdraw from the emotional experiences of one another. These effects could alter the functioning of societies, as they are conceived sociologically, potentially leading to decreases in social cooperation and the breakdowns of social connectedness [37].

\section{Limitations and Future Work}

While the results of the experiment were considered statistically significant, this study is unable to measure how long such effects may last-showing only immediate decreases in emotional acuity and it only contributes in terms of a single population-traditional college students, with a gender-biased makeup. This is of note as emotion recognition research has found significant differences across different age groups and genders [38].

It is also important to note that while impacts of cognitive dissonance have been widely replicated, there still might exist cultural differences; dissonance effects have been less successfully replicated in Japanese and Chinese cultures, for example [20]. While GERT-S is designed for use in various cultures, Phase 1 of the current experiment hinged on individual's ability to understand the words presentedindividual and cultural understandings of different words may change how participants identified tone or felt dissonance.

Furthermore, it should be noted that there were additional factors that may have potentially impacted participants throughout this study as the societal impacts of the COVID-19 pandemic were being increasingly felt around the world, and throughout participants' personal lives. This is what led to the shift of administration. Further research should seek to eliminate these limitations, reduce potential cultural bias, and replicate these results with larger groups and across different populations. 


\section{Competing Interests}

The authors declare that they have no competing interests.

\section{References}

1. Conley D (2017) You may ask yourself: An introduction to thinking like a sociologist (5th ed.). WW Norton \& Company.

2. Berkowitz AD (2003) Applications of social norms theory to other health and social justice issues. The social norms approach to preventing schoo and college age substance abuse: A handbook for educators, counselors, and clinicians.

3. Gross JJ, John OP (2003) Individual differences in two emotion regulation processes: Implications for affect, relationships, and well-being. J Pers Soc Psychol 85: 348-362.

4. Flynn JJ, Hollenstein T, Mackey A (2010) The effect of suppressing and not acceptingemotions on depressive symptoms: Is suppression different for men and women?. Personality and Individual Differences 49: 582-586.

5. Vogel DL, Heimerdinger-Edwards SR, Hammer JH, Hubbard A (2011) "Boys don't cry": Examination of the links between endorsement of masculine norms, self-stigma, and help-seeking attitudes for men from diverse backgrounds. J Couns Psychol 58: 368-382.

6. Festinger L (1962) A theory of cognitive dissonance (Vol. 2). Stanford university press.

7. Prasad J (1950) A comparative study of rumours and reports in earthquakes. British Journal of Psychology. General Section 41: 129-144.

8. Festinger L, Carlsmith JM (1959) Cognitive consequences of forced compliance. J Abnorm Psychol 58: 203-210.

9. Festinger L, Riecken H, Schachter S (1956) When prophecy fails: A social and psychological study of a modern group that predicted the destruction of the world. Lulu Press, Inc.

10. Auerbach $Y$ (1986) Turning-point decisions: A cognitive-dissonance analysis of conflict reduction in Israel-West German relations. Political Psychology 7: 533-550.

11. Beasley RK, Joslyn MR (2001) Cognitive dissonance and post-decision attitude change in six presidential elections. Political psychology 22: 521 540.

12. Axsom D, Cooper J (1985) Cognitive dissonance and psychotherapy: The role of effort justification in inducing weight loss. Journal of Experimental Social Psychology 21: 149-160.

13. Benozio A, Diesendruck G (2015) From effort to value: Preschool children's alternative to effort justification. Psychol Sci 26: 1423-1429.

14. Bullens L, van Harreveld F, Förster J, van der Pligt J (2013) Reversible decisions: The grass isn't merely greener on the other side; it's also very brown over here. Journal of Experimental Social Psychology 49: 1093-1099.

15. Frenkel OJ, Doob AN (1976) Post-decision dissonance at the polling booth Canadian Journal of Behavioural Science/Revue canadienne des sciences du comportement 8: 347-350.

16. Levy N, Harmon-Jones C, Harmon-Jones E (2018) Dissonance and discomfort: Does a simple cognitive inconsistency evoke a negative affective state? Motivation Science 4: 95-108.

17. Harmon-Jones E, Peterson H, Vaughn K (2003) The dissonance-inducing effects of an inconsistency between experienced empathy and knowledge of past failures to help: Support for the action-based model of dissonance. Basic and Applied Social Psychology 25: 69-78.

18. Nolan J, Nail P (2014) Further Evidence That Individuals With a High Preference for Consistency Are More Susceptible to Cognitive Dissonance. Psi Chi Journal of Psychological Research.

19. Hoshino-Browne E (2012) Cultural Variations in Motivation for Cognitive Consistency: Influences of Self-Systems on Cognitive Dissonance. Social and Personality Psychology Compass 6: 126-141.

20. Heine SJ, Lehman DR (1997) Culture, dissonance, and self-affirmation. Personality and Social Psychology Bulletin 23: 389-400.

21. Ekman P, Friesen WV (1969) The repertoire of nonverbal behavior: Categories, origins, usage, and coding. Nonverbal communication, interaction, and gesture.
22. Josephs IE (1994) Display rule behavior and understanding in preschool children. Journal of Nonverbal Behavior 18: 301-326.

23. Jones DC, Abbey BB, Cumberland A (1998) The development of display rule knowledge: Linkages with family expressiveness and social competence. Child development 69: 1209-1222.

24. Yagil D (2015) Display rules for kindness: Outcomes of suppressing benevolent emotions. Motivation and Emotion 39: 156-166.

25. Walsh G (2019) Service employees' naturally felt emotions: Do they matter? European Management Journal 37: 78-85.

26. Matsumoto D, Takeuchi S, Andayani S, Kouznetsova N, Krupp D, et al (1998) The contribution of individualism vs. collectivism to cross-national differences in display rules. Asian journal of social psychology 1: 147-165.

27. Diefendorff JM, Richard EM, Croyle MH (2006) Are emotional display rules formal job requirements? Examination of employee and supervisor perceptions. Journal of Occupational and Organizational Psychology 79: 273-298.

28. Moran CM, Diefendorff JM, Greguras GJ (2013) Understanding emotional display rules at work and outside of work: The effects of country and gender. Motivation and Emotion 37: 323-334

29. Santiago-Menendez M, Campbell A (2013) Sadness and anger: Boys, girls, and crying in adolescence. Psychology of Men \& Masculinity 14: 400-410.

30. McRae K, Ochsner KN, Mauss IB, Gabrieli JJ, Gross JJ, et al. (2008) Gender differences in emotion regulation: An fMRI study of cognitive reappraisal. Group Process Intergroup Relat 11: 143-162.

31. Karnilowicz HR, Waters SF, Mendes WB (2019) Not in front of the kids: Effects of parental suppression on socialization behaviors during cooperative parent-child interactions. Emotion 19: 1183-1191.

32. Lockwood PL, Seara-Cardoso A, Viding E (2014) Emotion regulation moderates the association between empathy and prosocial behavior. PloS one 9: e96555.

33. Hodges SD, Klein KJ (2001) Regulating the costs of empathy: the price of being human. The Journal of socio-economics 30: 437-452.

34. Cameron CD, Hutcherson CA, Ferguson AM, Scheffer JA, Hadjiandreou E, et al. (2019) Empathy is hard work: People choose to avoid empathy because of its cognitive costs. J Exp Psychol Gen 148: 962-976.

35. Schlegel K, Scherer KR (2016) Introducing a short version of the Geneva Emotion Recognition Test (GERT-S): Psychometric properties and construct validation. Behav Res Methods 48: 1383-1392.

36. Shamay-Tsoory SG, Aharon-Peretz J, Perry D (2009) Two systems for empathy: a double dissociation between emotional and cognitive empathy in inferior frontal gyrus versus ventromedial prefrontal lesions. Brain 132: 617-627.

37. Rawls J (1971) A theory of justice. Rawls. The Belknap.

38. Schlegel K, Grandjean D, Scherer KR (2014) Introducing the Geneva emotion recognition test: an example of Rasch-based test development. Psychol Assess 26: 666-672. 